Annuaire suisse de politique de développement

16 | 1997

Environnement et développement, 5 ans après Rio

\title{
Environnement, développement et coopération : enjeux et moyens d'action
}

Franco Romerio et Milad Zarin-Nejadan

\section{OpenEdition}

Édition électronique

URL : http://journals.openedition.org/aspd/804

DOI : 10.4000/aspd.804

ISSN : 1663-9669

Éditeur

Institut de hautes études internationales et du développement

\section{Édition imprimée}

Date de publication : 1 mars 1997

Pagination : 181-201

ISSN : 1660-5934

\section{Référence électronique}

Franco Romerio et Milad Zarin-Nejadan, «Environnement, développement et coopération : enjeux et moyens d'action », Annuaire suisse de politique de développement [En ligne], 16 | 1997, mis en ligne le 08 août 2012, consulté le 10 décembre 2020. URL : http://journals.openedition.org/aspd/804 ; DOI :

https://doi.org/10.4000/aspd.804 


\title{
ENVIRONNEMENT, DÉVELOPPEMENT ET COOPÉRATION : ENJEUX ET MOYENS D'ACTION
}

\author{
FRANCO ROMERIO ET MILAD ZARIN-NEJADAN*
}

\section{INTRODUCTION}

Jusqu'au début des années 1970, se préoccuper de la qualité de l'environnement passait souvent pour un luxe que ne pouvaient s'offrir les pays en développement. Lors de la Conférence des Nations Unies qui s'est tenue à Stockholm en 1972, le Premier Ministre indien Indira Gandhi s'est fait le porte-parole de bon nombre des pays en développement en déclarant : "Comment peut-on demander à ceux qui vivent dans les villages et les bidonvilles de préserver la pureté de l'air, des rivières et des océans quand leur propre vie est viciée ? La misère des pauvres et l'avidité des riches ne sont-elles pas les nuisances les plus graves ?" La préoccupation environnementale était alors considérée comme un obstacle au développement économique, en tout cas jusqu'à un certain stade.

Vingt ans après, en juin 1992, plus de 30'000 personnes se sont réunies à Rio de Janeiro pour assister à la Conférence des Nations Unies sur l'environnement et le développement (CNUED), baptisée « Sommet de la Terre ». Cette Conférence a rassemblé les représentants de plus de 180 nations, dont quelque 120 chefs d'Etat et de gouvernement, des organisations non gouvernementales (ONG), des industriels, des groupes religieux, des groupes ethniques, des éducateurs et des étudiants. Ce Sommet a été sans aucun doute un événement sans précédent par son ampleur, sa portée et les attentes qu'il a suscitées. On mesure ainsi tout le chemin parcouru depuis un quart de siècle par les thèses sur la compatibilité du développement économique et de la protection de l'environnement. La gestion saine de l'environnement et des ressources naturelles est désormais considérée, non plus comme un obstacle au développement, mais comme sa condition préalable, et constitue un élément clef de tout programme destiné à améliorer les conditions de vie des pauvres de la planète.

Cette contribution tente de faire le point sur les interactions multiples entre l'environnement et le développement économique, ainsi que sur les moyens concrets à mettre en œuvre dans le cadre des politiques de coopération et d'aide au développement afin d'atteindre des objectifs écologiques dans les pays du Sud. Elle est divisée en quatre parties. Après cette brève introduction, la partie 2 survolera les liens complexes et souvent ambigus existant entre l'environnement et le développement. Ensuite, la partie 3 passera en revue les divers moyens à disposition des pays développés et de la communauté internationale pour mettre en œuvre ou susciter des programmes d'assainissement écologique de caractère local ou global dans les pays du Sud. Enfin, la partie 4 tentera, en guise de conclusion, de faire le point sur les principaux enseignements que l'on peut tirer de cette analyse.

\footnotetext{
* Les auteurs tiennent à remercier Beat Bürgenmeier ainsi que le comité de rédaction de l'Annuaire pour leurs remarques et suggestions.
} 


\section{INTERACTIONS ENTRE ENVIRONNEMENT ET DÉVELOPPEMENT}

Dans cette partie, nous effectuerons un rapide survol des interactions multiples existant entre développement et environnement. ${ }^{1}$ La réflexion se situera délibérément à un niveau général, les problématiques soulevées étant souvent pertinentes aussi bien pour les pays développés que pour ceux en développement.

\subsection{PROBLÉMATIQUE ET ENJEUX}

Dans le passé, le développement économique et social a souvent été synonyme de dégradation de l'environnement. Cependant, on ne prenait pas sérieusement en considération l'hypothèse selon laquelle le développement pouvait provoquer de graves problèmes environnementaux au point de faire obstacle à sa poursuite. On avait en effet tendance à percevoir le problème surtout au niveau de la microéchelle ${ }^{2}$, car les dégâts se manifestaient surtout à cette échelle. Et même à ce niveau, on ne percevait que les problèmes les plus macroscopiques. Ceci était dû, d'une part, au manque de connaissances scientifiques, et d'autre part, à une culture qui accordait peu d'importance à l'environnement. Si les dégâts se manifestaient de manière suffisamment grave pour alerter l'opinion publique, on intervenait avec des mesures de type technique, législatif ou économique (plus rarement), ceci au moins dans les pays démocratiques. Mais aux yeux des responsables, ces problèmes ne représentaient pas plus que des « accidents de parcours ", qui pouvaient être résolus à l'aide de mesures ponctuelles. A cet égard, on peut mentionner le cas de la pollution de l'air à Londres, provoquée par la combustion du charbon dans les ménages et l'industrie, et en particulier la concentration de particules, qui au début du mois de décembre 1952 a été à l'origine d'une montée en flèche de la mortalité. Le gouvernement britannique a été obligé de mettre en place une législation en la matière, avec un certain succès. ${ }^{3}$ Une fois l'état d'urgence levé, il a fallu attendre les années 90 pour que le problème de la pollution par la concentration des particules soit remis à l'ordre du jour. ${ }^{4}$

Depuis un certain nombre d'années, on reconnaît qu'il existe des liens très étroits mais fort complexes entre environnement et développement. En effet, on a pris conscience de l'ampleur de la dégradation de l'environnement, du fait qu'elle n'a pas lieu seulement au niveau de la micro-échelle, mais également à ceux de la méso-échelle et de la macro-échelle et du fait de la gravité de ses effets sur la santé, la qualité de vie, l'économie et la société. On s'est rendu compte qu'à l'origine de nombreux problèmes il n'y avait pas simplement des dérapages ou des accidents de parcours, mais le processus du développement socio-économique lui-même. On sait désormais :

- que la dimension de l'environnement doit être intégrée pleinement dans le développement des pays industrialisés du Nord,

${ }^{1}$ Cf. Banque mondiale, Rapport sur le développement dans le monde 1992 - Le développement et l'environnement, Washington, 1992 ; et le numéro spécial de la Revue Tiers Monde ayant pour thème « Environnement et développement $»\left(\right.$ vol. 33, $\mathrm{n}^{\mathrm{O}}$ 130, 1992).

2 On distingue la micro-échelle (la pollution de l'air dans une ville, par exemple) de la méso-échelle (la mort des forêts) et de la macro-échelle (l'effet de serre).

3 Cf. Ministry of Housing and Local Government, Report of the Committee on Air Pollution (Beaver Report), Londres, HMSO, 1954.

4 Cf. A. Seaton et al., « Particulate Air Pollution and Acute Health Effects », The Lancet, 345, 21 janvier 1995. 
- que le développement devrait être repensé, car il ne suffit pas simplement d'ajouter l'environnement comme un nouveau paramètre au problème, et

- que ceci est d'autant plus urgent que les pays du Sud et les anciens pays communistes ont adopté le modèle de développement des pays industrialisés du Nord.

L'environnement et le développement sont dorénavant considérés comme deux composantes indissociables d'un même problème ; car un processus de développement qui délaisse les problèmes environnementaux et ne reconsidère pas les options du passé, non seulement se retournera contre lui-même à cause de la dégradation des conditions de vie et des bouleversements climatiques mais, faute de ressources et d'espaces où rejeter ses déchets, il finira par plafonner. La question fondamentale est celle des conséquences pour l'environnement et des limites au développement, surtout si la majorité de la population du globe s'aligne sur les modes de consommation des pays industrialisés et si ces derniers poursuivent leur croissance en délaissant la question environnementale. ${ }^{5}$

Le débat sur le "développement durable ", qui a eu un retentissement international lors de la CNUED en 1992, doit être situé dans cette perspective. ${ }^{6}$ La réflexion sur le développement durable tente de définir des modèles de développement économique et social de la planète qui soient en harmonie avec l'environnement. Elle reconsidère de manière plus ou moins explicite les options des modèles de développement du passé en essayant d'intégrer la dimension environnementale dans leurs mécanismes de fonctionnement. Elle adopte une optique de long terme qui prend en considération le sort des générations futures. Enfin, elle porte une attention particulière à ces masses humaines qui vivent dans la plus grande misère, mal nourries, mal logées, privées de soins médicaux et sans instruction.

\subsection{CROISSANCE ÉCONOMIQUE ET ENVIRONNEMENT'}

La croissance économique peut représenter une menace pour l'environnement. Elle peut accroître la charge sur l'environnement jusqu'à des limites intolérables. Toutefois, elle est en mesure d'engendrer plus de ressources pour financer la politique environnementale, les investissements dans les technologies propres, la réalisation des infrastructures nécessaires, etc. Par ailleurs, il serait illusoire de penser pouvoir affronter les principaux problèmes environnementaux actuels dans une situation de stagnation économique, à moins d'accepter des changements radicaux dans l'affectation des ressources, tels que la réduction des dépenses militaires au profit de la politique environnementale. ${ }^{8}$ Notons que la croissance économique rend aussi le climat politique plus favorable à la politique

5 Cf. B. Giovannini, « Les menaces sur la planète », CADMOS - Cahiers trimestriels du Centre Européen de la Culture, vol. $13, \mathrm{n}^{2} 49,1990$.

${ }^{6}$ Parmi les multiples définitions de développement durable, on retiendra la suivante : «Sustainable development involves devising a social and economic system which ensures [...] that real incomes rise, that educational standards increase, that the health of the nation improves, that the general quality of life is advanced ».

7 Cf. D. Pearce, A. Markandya et E. Barbier, Blueprint for a Green Economy, Londres, Earthscan, 1992, pp. 1-2.Cf. G. M. Grossman et A. B. Krueger, Economic Growth and the Environment, Cambridge University Press, 1994.

8 Les pays nouvellement industrialisés de l'Asie, qui connaissent actuellement une forte croissance économique (taux de croissance réels de 6-7\%), disposent quant à eux d'un potentiel supérieur aux autres pays du Sud pour financer les investissements dans l'environnement. Ils peuvent aussi tirer profit de l'expérience des pays occidentaux. 
environnementale. En effet, pendant les périodes de récession, d'autres problèmes comme le chômage priment la préoccupation environnementale.

Pour qu'une synergie positive puisse se réaliser entre la croissance économique et l'environnement, deux conditions doivent être réunies :

- l'existence d'un éventail de mesures de type juridique, technique et économique permettant de minimiser l'impact de la croissance économique sur l'environnement tout en garantissant le respect des différentes limites ;

- l'utilisation d'une partie des ressources générées par la croissance économique pour financer les mesures de lutte contre la dégradation de l'environnement.

Les instruments économiques sont particulièrement importants, car ils permettent de réaliser un arbitrage entre les avantages de la réduction de l'impact environnemental d'une activité donnée et les coûts qu'elle entraîne. Par exemple, dans le cas d'une activité industrielle émettant des substances polluantes dans l'atmosphère, l'internalisation des coûts environnementaux permet d'obtenir un prix de marché qui reflète à la fois les coûts privés supportés par les entreprises, les coûts environnementaux supportés par la communauté et les préférences des consommateurs.

Le problème du financement de la protection de l'environnement ne peut pas être évoqué sans rappeler la question de l'efficacité avec laquelle on utilise les ressources disponibles. En règle générale, les premières mesures ont tendance à être les plus efficaces : investir 1 million de francs pour réduire la pollution d'une centrale thermique de conception ancienne aura un impact nettement plus important que le même montant investi dans une centrale moderne à cycle combiné. Dès lors, il ne fait pas de doute que, dans certains domaines, il serait plus efficace d'utiliser les ressources consacrées à la lutte contre la dégradation de l'environnement dans les anciens pays communistes et dans les pays du Sud plutôt que dans les pays industrialisés.

\subsection{POLITIQUE ENVIRONNEMENTALE ET CROISSANCE ÉCONOMIQUE}

On peut distinguer les mesures de lutte contre la dégradation de l'environnement, entre autres, d'après leur impact sur la croissance économique. Il y a d'abord les mesures qui sont positives à la fois du point de vue environnemental et économique. Citons à cet égard la suppression de différents types de subventions (subventions pour des intrants énergétiques, des engrais et pesticides, par exemple) qui représentent une source de distorsions pour l'économie ${ }^{9}$ tout en encourageant l'utilisation de matières premières polluantes, ou encore les investissements pour l'approvisionnement en eau salubre ou dans la protection des sols. D'autre part, il y a les mesures qui ne sont profitables du point de vue économique que si l'on prend en considération les coûts environnementaux qui résulteraient de l'inaction, comme par exemple l'introduction d'une taxe sur les émissions de $\mathrm{CO}_{2}$, ou les investissements dans des équipements permettant la réduction des émissions dans l'atmosphère par les industries et les centrales thermiques fossiles. Ce sont ces dernières mesures qui soulèvent le plus d'opposition. En situation de crise

9 Par « distorsion », on entend une mauvaise utilisation des ressources productives (capital naturel, capital manufacturé, capital humain) provoquée par le fait que le prix d'un bien ne reflète pas son coût marginal. 
économique ou de sous-développement on préfère souvent renvoyer ces considérations sine die.

La production et la commercialisation de technologies respectueuses de l'environnement représentent potentiellement un créneau porteur d'avenir pour l'industrie. Toutefois, jusqu'ici les efforts déployés dans ce sens sont restés en dessous des attentes. Cela peut être expliqué par le fait que les incitations économiques et les réglementations en matière environnementale sont encore relativement peu développées et trop limitées à certaines régions du monde.

\subsection{MARCHÉ ET ENVIRONNEMENT}

La problématique de la politique environnementale pose implicitement la question du fonctionnement du marché, autrement dit cette «main invisible » qui, sans certaines défaillances, garantirait l'allocation optimale des ressources. On peut relever trois lacunes du marché en relation avec l'environnement :

- Les prix des produits ne reflètent pas les coûts et les bénéfices externes. Autrement dit, les prix sont calqués sur les coûts privés et ne prennent pas en considération les coûts et les bénéfices sociaux. A l'origine de cette défaillance, il y a l'absence de droits de propriété. Lorsqu'on a librement accès aux ressources environnementales, comme la mer et l'atmosphère, on ne paie pas le prix de leur utilisation et on a naturellement tendance à en abuser.

- Dans certaines circonstances, les agents économiques privés peuvent privilégier le court terme alors que les problèmes environnementaux nécessitent souvent une vision de long terme. Une sorte de « hiatus » peut donc s'instaurer entre les solutions offertes par le marché et les exigences de l'environnement. Prenons le cas des investissements en recherche et développement (R\&D) de technologies respectueuses de l'environnement pour la deuxième moitié du siècle prochain. Vraisemblablement, il s'agit là d'un horizon trop éloigné pour la plupart des entrepreneurs. Or, il se peut qu'il soit nécessaire d'investir des sommes considérables en $\mathrm{R} \& \mathrm{D}$ dès maintenant pour disposer de technologies économiquement viables dans cinquante ans.

- La prise en compte de l'incertitude par les agents privés n'est pas toujours optimale du point de vue de l'environnement. Certes, une attitude « risquophobe » de la part de ces agents peut s'avérer favorable à l'environnement, dans la mesure où elle conduit à l'abandon de technologies, procédés ou produits pouvant avoir des coûts environnementaux très élevés (pour l'entrepreneur il y a le coût du dédommagement des victimes). Cependant, ce type d'attitude peut aussi conduire à l'abandon de technologies qui, si certains états de la nature se réalisent, peuvent se révéler payantes à la fois du point de vue économique et environnemental. Par exemple, l'option d'investir en R\&D des technologies permettant l'abandon du pétrole et du charbon ne sera rentable du point de vue économique que si les scénarios pessimistes sur les conséquences du réchauffement de la Terre se réalisent. 


\subsection{RECOMMANDATIONS DE POLITIQUE ÉCONOMIQUE}

Il convient de mentionner des solutions permettant d'inclure la dimension environnementale dans le processus de développement afin d'intégrer celui-ci à un schéma et développement durable. Les recommandations de politique économique qui suivent se réfèrent aux problèmes des externalités d'une part, et de l'horizon temporel et de son incertitude d'autre part.

Concernant la prise en compte des externalités environnementales, cinq impératifs doivent être rappelés : ${ }^{10}$

- Les droits de propriété devraient être clarifiés partout où ceci est nécessaire et faisable. A cet égard, on peut relever que les systèmes de propriété collective selon lesquels ce sont les communautés qui établissent les règles sur les conditions d'accès et d'utilisation - permettent aussi d'obtenir une gestion rationnelle des ressources naturelles (pâturages, forêts, réseaux d'irrigation, lieux de pêche, etc.).

- Les coûts externes devraient être internalisés. Dans beaucoup de cas, toutefois, l'estimation de ces coûts soulève des problèmes extrêmement compliqués. Ẹn effet, comment estimer les coûts externes de la destruction de la couche d'ozone ou d'un accident nucléaire, alors que les cancers et les défauts génétiques peuvent apparaître un demi-siècle plus tard ? Dans bien des cas, il est probable que l'on soit obligé de se tourner vers un autre type d'approche, à savoir la fixation du prix d'un bien engendrant un certain type de pollution à un montant suffisamment élevé pour en limiter la consommation (et donc la pollution) au niveau admis («prix d'évitement»).

- Les projets ayant des effets environnementaux plus ou moins sensibles, devraient faire l'objet d'une évaluation du type coûts-bénéfices (ou une méthode analogue telle que coûts-avantages). Ceci permettrait aux décideurs de prendre en considération les retómbées environnementales et sociales de la réalisation d'un projet, comme la disparition de zones habitées et de biotopes particulièrement précieux dans le cas de la construction d'un barrage, ou la formation des travailleurs et la protection des sols dans le cas d'un projet visant à rationaliser l'exploitation agricole d'une région.

- L'internalisation des coûts et les études d'impact devraient prendre soigneusement en considération les risques d'origine naturelle, comme les tremblements de terre, les éruptions volcaniques ou les inondations, et les risques technologiques, qui concernent tout particulièrement les industries chimique et nucléaire. En effet, certaines catastrophes, dont la probabilité de survenance peut être très faible, peuvent être lourdes de conséquence pour l'environnement et le développement socio-économique d'une région. Il suffit de rappeler que l'accident qui s'est produit dans l'usine chimique de Bhopal en Inde en 1984 a fait 2'500 morts et des milliers de blessés, et que le tremblement de terre au Salvador en 1986 a provoqué des pertes de l'ordre de $25 \%$ de son PNB et de $40 \%$ de sa dette extérieure. ${ }^{11}$

${ }^{10}$ Cf. P. S. Dasgupta et G. M. Heal, Economic Theory and Exhaustible Resources, Cambridge University Press, 1979 ; OCDE, Gérer l'environnement: le rôle des instruments économiques, Paris, OCDE, 1994 ; et R. Sugden et A. Williams, The Principles of Practical Cost-Benefit Analysis, Oxford University Press, 1978.

${ }^{11}$ Cf. C. Clarke et M. Munasinghe (eds.), Disaster Prevention for Sustainable Development, Washington, US National Academy of Sciences, Banque mondiale, à paraître. 
- Plus généralement, il convient de souligner l'importance de certains outils d'analyse à cet égard, notamment la comptabilité environnementale dans les entreprises, les analyses du cycle de vie des produits et l'intégration de l'environnement dans les comptes nationaux.

Concernant les problèmes liés à l'horizon temporel et à la gestion de l'incertitude, nous nous contenterons de relever l'enjeu auquel est confrontée la politique environnementale. ${ }^{12}$ Celle-ci doit mettre en place un ensemble de mesures qui soit :

- suffisamment flexible pour qu'on puisse le corriger au fur et à mesure que de nouvelles informations apparaissent et que les marges d'incertitude diminuent ;

- suffisamment équilibré (ou, si l'on veut, en rapport avec les bénéfices escomptés) pour éviter de dépenser trop ou trop peu d'argent, avec le risque de gaspiller des ressources, ou bien de payer un jour très cher le prix de l'inaction d'aujourd'hui ; et finalement

- suffisamment efficace pour éviter le déclenchement de processus irréversibles.

Ces remarques illustrent la complexité de la prise de décision en avenir incertain. D'une part, il faut opérer un arbitrage entre court terme et long terme, et entre différents états de la nature qui pourraient ou non se réaliser. D'autre part, il faut prendre en considération le fait qu'en matière environnementale on a souvent affaire à des phénomènes irréversibles. La meilleure illustration de ce problème est fournie par les difficultés rencontrées lorsqu'on essaie de définir des stratégies de lutte contre l'effet de serre.

\section{Mesures incitatives et réglementaires destinées à la protection de l'environnement ${ }^{13}$}

\begin{tabular}{|c|c|c|c|}
\hline Type de mesure & & Variable affectée & \\
\hline & Prix & Quantité & Technologie \\
\hline \multicolumn{4}{|l|}{ Incitations } \\
\hline Directes & $\begin{array}{l}\text { Redevance de déversement, } \\
\text { taxes d'abattage, } \\
\text { systèmes de consignation }\end{array}$ & $\begin{array}{l}\text { Droits d'émission } \\
\text { négociables, droits } \\
\text { de pêche négociables }\end{array}$ & $\begin{array}{l}\text { Taxes sur les } \\
\text { émissions } \\
\text { présumées }\end{array}$ \\
\hline Indirectes & $\begin{array}{l}\text { Taxes sur les } \\
\text { carburants, caution de bon } \\
\text { fonctionnement }\end{array}$ & $\begin{array}{l}\text { Droits négociables } \\
\text { applicables aux mo- } \\
\text { yens de production } \\
\text { ou à la production }\end{array}$ & $\begin{array}{l}\text { Subventions de } \\
\text { R\&D et de } \\
\text { rendement } \\
\text { énergétique }\end{array}$ \\
\hline \multicolumn{4}{|l|}{ Réglementations } \\
\hline Directes & - & $\begin{array}{l}\text { Normes d'émission, } \\
\text { quotas d'abattage }\end{array}$ & $\begin{array}{l}\text { Normes techniques } \\
\text { obligatoires }\end{array}$ \\
\hline Indirectes & - & $\begin{array}{l}\text { Zonage, interdiction } \\
\text { et contingentement } \\
\text { des produits et des } \\
\text { moyens de production }\end{array}$ & $\begin{array}{l}\text { Normes de rende- } \\
\text { ment pour les mo- } \\
\text { yens de production } \\
\text { tou les procédés }\end{array}$ \\
\hline
\end{tabular}

${ }_{12}^{12}$ Cf. R. S. Pyndick, « Irreversibility, Uncertainty and Investment », Journal of Economic Literature, vol. 29, nº 3, 1991.

${ }^{13}$ Source : Banque mondiale, op. cit., 1992, p. 77. 
Le tableau recense quelques mesures de politique environnementale du type économique et juridique utilisées dans les pays industrialisés. Notons que dans certains domaines (santé, par exemple) de bons résultats peuvent être obtenus à travers l'information et l'éducation sans qu'il soit nécessaire d'agir au moyen de ce type d'instruments. Le tableau suscite les commentaires suivants :

- Une distinction est faite entre les mesures incitatives qui en général agissent à travers le mécanisme du marché (« incitations par le marché »), et les mesures réglementaires (« direction et commandement ») qui ont un caractère plus dirigiste. ${ }^{14}$ Ces dernières deviennent absolument indispensables lorsqu'on a affaire à des atteintes potentielles à l'environnement extrêmement graves : déchets toxiques, matières radioactives, etc. Chronologiquement, les mesures réglementaires ont précédé les mesures incitatives. Jusqu'à une période récente, on cherchait surtout à endiguer la pollution par des mesures réglementaires. Depuis peu, on cherche également à la prévenir par des mesures incitatives. Une des difficultés de la politique environnementale réside dans le fait de trouver un bon équilibre entre ces deux types de mesures.

- Toutes ces mesures, avant d'être adoptées, doivent être évaluées non seulement du point de vue de l'allocation des ressources et de leurs effets sur l'environnement, mais aussi sous l'angle de leurs effets sociaux, en particulier sur les couches les plus défavorisées de la société (si nécessaire, des mesures d'accompagnement pour alléger l'impact sur les pauvres peuvent être envisagées).

- Il faut souligner l'importance de l'harmonisation internationale des politiques environnementales, pour éviter l'exportation des activités polluantes et à risque dans les pays qui sont moins exigeants à cet égard.

- Enfin, il ne faut pas perdre de vue l'importance de la lutte contre les comportements criminels (comme la création de dépôts sauvages de déchets dangereux) et contre la corruption, qui représentent des obstacles de taille pour la mise en œuvre efficace de la politique environnementale.

\section{LA COOPÉRATION AU DÉVELOPPEMENT FACE AUX ENJEUX ÉCOLOGIQUES}

Dans cette partie, nous passerons en revue un certain nombre d'instruments pouvant être utilisés pour intégrer la dimension environnementale dans les politiques d'aide au développement des pays industrialisés, dont la Suisse, et des organismes internationaux de coopération au développement. Nous nous concentrerons sur les instruments à effet relativement direct et laisserons de côté ceux ayant un effet indirect sur l'environnement, par exemple par le biais de l'éducation, la santé ou la maîtrise démographique.

\subsection{ACCENTUATION ÉCOLOGIOUE DE L'AIDE BILATÉRALE ET MULTILATÉRALE}

Les objectifs environnementaux à caractère local ou global peuvent être réalisés en partie par l'intégration ou le renforcement de dimensions écologiques dans les programmes existants d'aide bilatérale et multilatérale. L'aide publique au développement peut porter spécifiquement sur des projets environnementaux. Comme

${ }^{14}$ Dans une acception plus large des mesures réglementaires, on pourrait inclure la définition des droits de propriété. 
nous l'avons déjà souligné précédemment, l'amélioration des conditions environnementales peut influencer positivement la croissance économique des pays en développement et résulter en un «double-dividende ». Notons qu'un certain nombre de projets de développement financés par la Suisse comportent déjà un accent écologique très marqué. Parmi les projets de nature agricole on peut citer $:^{15}$ - le programme forestier visant une exploitation durable des forêts à Madagascar,

- la plantation d'arbres dans les rizières du nord-ouest du Bangladesh,

- l'amélioration de la fertilité des sols dans les régions arides et montagneuses au Népal,

- la lutte biologique contre les parasites touchant le manioc en Afrique, et

- l'augmentation de la production dans les zones extrêmement sèches habitées par les nomades du Sahel.

Nul doute que les projets de développement à effet environnemental direct seront de plus en plus nombreux dans les années à venir.

Cela dit, à moins que le volume d'aide publique au développement n'augmente significativement dans un proche avenir, ce qui est peu probable vu les difficultés financières des collectivités publiques dans la plupart des pays développés y compris la Suisse, la réalisation des projets environnementaux en puisant dans les enveloppes budgétaires actuelles consacrées à l'aide au développement risque de se faire au détriment d'autres projets tout aussi importants sur le plan du développement. Donc, la marge de manœuvre pour la réalisation des projets environnementaux au Sud dans le cadre des programmes d'aide existants paraît limitée. Ce qui est par contre beaucoup plus facilement envisageable, c'est d'intégrer les préoccupations environnementales dans l'évaluation des divers projets de développement considérés. L'idée de prendre en compte les externalités dans l'analyse coûts-avantages précédant la décision de financer ou non un projet fait petit à petit son chemin dans les programmes d'aide au développement des pays industrialisés occidentaux. En Suisse, ce type d'étude d'impact n'est malheureusement pas encore institutionnalisé.

Les organismes internationaux tels que la Banque mondiale ont intégré, dès les années quatre-vingt, ces préoccupations dans leurs processus de décision du financement de projets dans les pays en développement. ${ }^{16}$ C'est en 1987 , suite à une série de critiques émises à l'égard d'un certain nombre d'opérations de prêts, telles que le projet de Polonoroeste au Brésil, le projet du développement de l'élevage au Botswana et le projet de transmigration en Indonésie, que la Banque mondiale a décidé de réviser fondamentalement sa politique pour accorder une grande importance à la gestion de l'environnement. Elle a ainsi mis en œuvre une série d'activités au premier rang desquelles viennent des rapports par pays sur des problèmes d'environnement, complétés au besoin par des études à portée régionale. Les résultats de ces recherches sont intégrés dans les études sectorielles et les analyses économiques par pays, sur lesquels la Banque s'appuie pour mener des opérations de prêt et fixer des priorités dans le dialogue qu'elle entretient avec les gouvernements sur leur politique économique.

\footnotetext{
${ }^{15}$ Voir à ce propos Message du 20.4.1994 concernant la continuation de la coopération technique et de l'aide financière en faveur des pays en développement, pp. 112-120.

${ }^{16}$ Cf. J. Warford et Z. Partow, «Evolution de la politique environnementale de la Banque mondiale », Finances et Développement, décembre 1989.
} 
Les programmes d'ajustement structurel (PAS) constituent un autre instrument à disposition de la communauté internationale pour atteindre des objectifs environnementaux de nature globale dans les pays en développement. En raison de dérèglements réels dans les domaines financier, budgétaire et monétaire, en raison aussi de la crise de la dette et de la gestion de son remboursement, un nombre très important des pays en développement, en particulier en Afrique, en Amérique latine et en Europe de l'Est ont adopté des PAS élaborés par le Fonds monétaire international en vue de restaurer leurs équilibres extérieurs, budgétaires et monétaires. L'impact environnemental de ces programmes peut être plus ou moins direct. D'abord, dans la mesure où la mise en œuvre de ces programmes conduit à des améliorations significatives dans des domaines tels que la santé, l'éducation ou la démographie, les retombées écologiques ont de fortes chances d'être positives sinon immédiates. Ensuite, étant donné que la plupart de ces programmes visent à rétablir la « vérité des prix » dans les pays en développement, ils exercent également des effets écologiques plus directs, notamment au travers de la réduction voire la suppression des subventions étatiques au prix de l'énergie.

Notons toutefois que les PAS sont souvent difficiles à mettre en œuvre et leur impact environnemental pourrait dans certains cas s'avérer néfaste. En effet, diverses critiques des PAS ont relevé que ceux-ci, en favorisant la promotion des exportations, peuvent contribuer à accélérer l'exploitation de certaines ressources naturelles (bois tropicaux par exemple) dans des conditions non conformes au développement durable.

\subsection{CONVENTIONS INTERNATIONALES}

Les négociations internationales constituent un moyen relativement "peu coûteux " d'intervention en matière d'environnement à disposition des pays développés et des organisations internationales. La première convention sur l'environnement de portée globale a été le Protocole de Montréal relatif aux substances qui appauvrissent la couche d'ozone, qui date de $1987 .{ }^{17}$ Les parties contractantes du Protocole de Montréal se sont engagées, d'une part, à réduire progressivement de moitié, jusqu'à l'an 2000, leur production de composés chimiques risquant de dégrader la couche d'ozone stratosphérique et, d'autre part, à stopper la progression des halons sur les marchés commerciaux. Vu les inquiétudes provoquées par l'apparition d'éléments nouveaux concernant l'ampleur de la destruction de la couche d'ozone et la rapidité de sa disparition, le Protocole de Montréal a subi une révision profonde en juin 1990. Il stipule désormais que les chlorofluorocarbures (CFC), halons et tétrachlorures de carbone seront totalement interdits d'ici l'an 2000, alors que quelques autres substances déployant les mêmes effets ravageurs le seront d'ici à 2005. Cet amendement a été accompagné du lancement d'un Fonds pour l'ozone qui doit permettre aux pays en développement de remplir les engagements auxquels cet accord les lie.

Dans ce contexte, l'événement le plus marquant de ces dernières années a été la CNUED en 1992 à Rio. Or, compte tenu des espoirs qui avait été placés en elle,

${ }^{17}$ Cf. Office fédéral de l'environnement, des forêts et du paysage, Rapport sur l'état de l'environnement 1993, Berne, OFEFP, 1994, p. 96. 
la Conférence est aujourd'hui qualifiée de relativement décevante dans la mesure où elle n'a débouché sur aucun engagement précis quant au calendrier des mesures à prendre ou au renforcement des programmes d'aide. Néanmoins, la Conférence a adopté différents documents finals et a abouti à la signature de deux conventions. La Déclaration de Rio sur l'environnement et le développement, l'Agenda 21 et les Principes cadres sur la protection des forêts ne revêtent aucune obligation juridique. La Convention sur les changements climatiques et la Convention sur la diversité biologique sont en revanche juridiquement contraignantes et doivent être ratifiées par les gouvernements avant de pouvoir entrer en vigueur.

La Déclaration de Rio est un bref exposé de principes fondamentaux destinés à guider le comportement humain à l'égard de l'environnement en définissant les droits et les responsabilités des pays, des collectivités et des particuliers. Elle est une version moins ambitieuse de la Charte de la Terre initialement envisagée. L'idée d'une Charte de la Terre, soutenue par la Suisse, dont l'importance aurait été comparable à celle de la Déclaration universelle des droits de l'homme n'a pas été retenue au Sommet de Rio à cause des divergences apparues au niveau de priorités des pays industrialisés et des pays en développement. Les premiers mettaient davantage l'accent sur l'écologie et la responsabilité commune envers l'environnement global alors que les seconds voulaient mettre au premier plan la problématique du développement. Quant à l'Agenda 21, il s'agit d'un ambitieux catalogue contenant un plan d'action de 800 pages pour le $\mathrm{XXI}^{\mathrm{e}}$ siècle et portant sur plus de 100 domaines d'action. Les premiers chapitres traitent des aspects sociaux et économiques d'un développement durable, notamment de stratégies pour lutter contre la pauvreté et pour la santé de la population, mais la majeure partie du document concerne des problèmes spécifiques d'environnement. Notons que chaque pays signataire s'est engagé à mettre en œuvre l'Agenda 21 au niveau national. En Suisse, un groupe de coordination interdépartemental a été chargé de préparer un plan national d'action. Enfin, les Principes cadres sur la protection de la forêt sont une simple recommandation pour accélérer les programmes de reboisement, de plantations forestières et de préservation des forêts et pour mieux tenir compte des droits des habitants et des pays d'accueil dans le partage du produit d'exploitation des forêts.

La Convention sur les changements climatiques, de caractère contraignant, est un des acquis majeurs de la CNUED. Elle vise à stabiliser les concentrations de gaz à effet de serre, principalement le gaz carbonique. Les pays industriels reconnaissent qu'il est souhaitable de ramener, d'ici l'an 2000, ces émissions à leurs niveaux de 1990. Ratifiée par 120 pays, dont la Suisse, cette Convention est entrée en vigueur le 21 mars 1994. La première réunion de la Conférence des Parties à la Convention s'est déroulée à Berlin en mars-avril 1995. Si les Etats membres ont reconnu l'insuffisance de l'objectif fixé pour l'an 2000, aucun accord n'a pu être trouvé sur des mesures à prendre au-delà de cette date. Dans une phase pilote, les pays qui le souhaitent pourront conjointement baisser les émissions de gaz à effet de serre. Les débats comprenaient des enjeux Nord-Sud importants, concernant notamment les responsabilités respectives des pays industrialisés et des pays en développement ainsi que le transfert de technologies plus propres. Les uns comme les autres sont toutefois loin de constituer des blocs soudés. Certains pays industrialisés, dont la Suisse, sont prêts à faire plus dans ce domaine que certains autres, tels les Etats-Unis. Parmi les pays en développe- 
ment, on trouve à un extrême les petits pays insulaires, qui exigent des mesures plus contraignantes et plus radicales, et à l'autre extrême les pays exportateurs de pétrole, opposés aux mesures limitant l'utilisation des énergies fossiles.

Enfin, la Convention sur la diversité biologique constitue l'autre acquis de la CNUED. Aux termes de cette Convention, les pays doivent adopter des règlements pour préserver leurs ressources biologiques. Elle rend les nations juridiquement responsables du comportement de leurs entreprises privées dans d'autres pays et recommande qu'un appui technique et une indemnisation soient fournis aux pays en développement pour le prélèvement de matériels génétiques. Cependant, la Convention reste floue sur les modalités d'application de ces principes. On s'oriente donc vers un système d'accords bilatéraux ou commerciaux. ${ }^{18}$ Cette Convention a été signée à Rio par 156 pays, dont la Suisse.

Les négociations internationales en matière d'environnement souffrent de la lenteur des processus de négociation et de la fragilité de leurs résultats. ${ }^{19}$ En effet, mis à part le Protocole de Montréal dont la rapidité de conclusion est largement liée à la pression des scientifiques et à la disponibilité des entreprises, l'expérience montre que les négociations à but environnemental, qu'elles se passent au niveau régional ou au nivéau international, sont extrêmement longues et complexes. Il n'est pas rare qu'une décennie ou deux soient nécessaires pour arriver à la conclusion de conventions ou de traités en la matière. Cela a été le cas du droit de la mer, des négociations sur les grands fleuves ou de celles sur le changement climatique et la biodiversité, qui se sont révélées longues et complexes. Les raisons de cette lenteur tiennent à la fois à la complexité des causes qui interfèrent dans la négociation, et qui n'ont pas toujours un lien direct avec l'environnement, mais également à la culture des négociateurs eux-mêmes et aux conditions et possibilités, en particulier financières, de la mise en œuvre des conventions et traités. Dès lors, il apparaît que la connaissance scientifique des processus de négociation et la formation des négociateurs dans les pays en développement constituent une priorité majeure dans le domaine des sciences sociales appliquées à la relation environnement-développement. Il s'agit, entre autres, de faciliter la connaissance des grands dossiers techniques, juridiques et financiers internationaux et de permettre ainsi l'accélération des processus de négociation. Notons que l'Académie internationale de l'environnement, basée à Genève et financée en partie par la Confédération, reconnaît l'importance de cet aspect du problème en déployant un effort particulier pour la formation des négociateurs en provenance des pays du Sud.

\subsection{POLITIQUE COMMERCIALE}

Le commerce international peut avoir des effets divers sur l'environnement. ${ }^{20}$ Premièrement, le commerce peut inciter les activités de production à se déplacer des zones où l'environnement est plus fragile vers des zones où la productivité peut être plus durable, et vice versa. Deuxièmement, la libéralisation des

\footnotetext{
${ }^{18}$ On peut citer comme exemple l'accord conclu entre la multinationale pharmaceutique Merck et l'Institut national de la biodiversité du Costa Rica. Voir à ce propos « Biodiversité au Costa Rica - INBIO veritas », Solidaire, n 121, février 1994.

${ }^{19}$ Cf. A. Benachenhou, « Environnement et développement », Revue Tiers Monde, vol. 23, n² 130, avril-juin 1992.

${ }^{20} \mathrm{Cf}$. FAO, « Le commerce agricole à l'aube d'une ère nouvelle », Rapport 1995, Rome, FAO, 1995.
} 
échanges modifie la structure et le volume de la consommation, de la production et des revenus mondiaux, ce qui peut avoir sur l'environnement des effets allant bien au-delà du déplacement de la consommation et de la production d'un pays à l'autre. Troisièmement, le commerce influe sur le développement économique et crée de nouveaux créneaux pour une utilisation rentable des ressources productives. Par exemple, les exportations de produits agricoles sont, pour beaucoup de pays, une source très importante de devises.

Remarquons également que, quand les revenus augmentent, la demande en ressources naturelles grandit, entre autres à cause de l'accroissement de la mobilité, ce qui est nocif pour l'environnement. Mais cette augmentation de revenus peut aussi se traduire par une plus grande exigence quant à la qualité de l'environnement. Parallèlement, les investissements dans la conservation des ressources naturelles sont à la fois plus faciles à réaliser et mieux accueillis par la population. De plus, en admettant que la hausse de revenus s'accompagne d'une baisse du taux de croissance démographique, la pression exercée sur les ressources naturelles diminue. Lorsque les revenus augmentent et que l'emploi s'améliore, l'éventail des possibilités s'élargit et les populations rurales sont moins repoussées vers les zones écologiquement fragiles, comme les versants abrupts des montagnes.

On constate donc que les liens entre le commerce, le développement et l'environnement sont extrêmement complexes. Durant le dernier cycle des négociations du GATT (Uruguay Round), plusieurs études, souvent centrées sur l'agriculture et l'exploitation des ressources naturelles, ont été effectuées sur l'impact environnemental de la libéralisation multilatérale des échanges. ${ }^{21}$ L'impression générale issue de ces analyses est que, dans les économies développées, l'environnement bénéficiera globalement d'une réduction des distorsions affectant les marchés agricoles du fait de la diminution du caractère intensif de l'agriculture, qui se traduit par une moindre utilisation d'engrais et de pesticides, par la reconversion des terres à d'autres usages, par la réduction de la dimension des exploitations ou par des cessations d'activité dans l'agriculture. Quant aux économies du Sud, elles enregistreraient plutôt des évolutions en sens inverse à la suite de la libéralisation des échanges. Dans l'agriculture, le recours à des méthodes plus intensives (mécanisation, utilisation des engrais et de pesticides) avec ses effets en termes d'utilisation et de pollution des eaux, d'érosion et d'extension des surfaces cultivées est à prévoir lorsque la libéralisation se traduit par une amélioration des termes de l'échange. Sans vouloir généraliser ces résultats à l'ensemble des domaines touchés par la libéralisation commerciale, on peut d'ores et déjà émettre de sérieux doutes quant à l'effet bénéfique du commerce sur l'environnement dans les pays en développement.

Les instruments de politique commerciale constituent néanmoins un moyen relativement simple et peu coûteux d'inciter les pays en développement à adopter des standards de consommation et des méthodes de production moins nocifs pour l'environnement. Il s'agit en fait d'amener ces pays à adopter les normes occidentales supposées plus écologiques. Cela peut être réalisé de manière plus ou moins douce. D'abord, les pays en développement peuvent être encouragés à internaliser dans une certaine mesure les coûts environnementaux par la simple

\footnotetext{
${ }^{21}$ Pour une liste complète de ces études, voir J. Beghin, D. Roland-Holst et D. van der Mensbrugghe, « Etude de la relation entre commerce et environnement : dimensions globales ", Revue économique de l'OCDE, $\mathrm{n}^{2}$ 23, hiver 1994.
} 
modification des conditions dans lesquelles ils exportent leurs produits (voir plus loin). Mais, le commerce international peut également avoir des effets bénéfiques plus directs sur l'environnement. Une étude récente de la Conférence des Nations Unies sur le commerce et le développement (CNUCED) montre clairement que les échanges commerciaux contribuent à la diffusion des standards pro-environnementaux. ${ }^{22}$ Par exemple, les exigences environnementales des grands marchés internationaux incitent les pays en développement à améliorer les standards de production et les réglementations sur leurs marchés domestiques.

Les instruments commerciaux peuvent aussi exercer un impact sur la protection de l'environnement dans les pays en développement de manière plus coercitive. En effet, les règles du GATT permettent aux pays de fixer leurs propres standards écologiques (article XX) ${ }^{23}$ Les pays ont ainsi les moyens d'interdire l'importation des produits ne correspondant pas à une certaine norme environnementale. Ainsi, les produits alimentaires contenant des résidus de pesticide ou les jouets montrant des traces de toxines dans la peinture peuvent être interdits d'accès au territoire national. En faisant respecter ces standards nationaux, les pays doivent seulement faire preuve d'égalité de traitement à l'égard des produits nationaux et étrangers. La question est de savoir où s'arrête le souci écologique légitime et où commence le protectionnisme déguisé. Ces dernières années quelques différends révélateurs ont surgi à propos de ces standards écologiques. Parmi les multiples désaccords commerciaux de nature environnementale on peut citer ceux sur les réglementations concernant l'emballage de boissons ou encore le contenu minimal en fibre recyclée de certains produits à base de papier. ${ }^{24}$

Notons que les règles du GATT n'admettent pour l'instant que les exigences environnementales en aval portant sur les produits eux-mêmes et non encore sur les procédés de production en amont. Or, les défenseurs de l'environnement s'intéressent également et de plus en plus aux externalités liées à la production, telles que la pollution, l'efficience énergétique et la gestion des déchets. Les pays développés pourraient également vouloir se protéger contre ce qu'il est convenu d'appeler l'éco-dumping, ou la « subvention environnementale », censé procurer un avantage comparatif déloyal aux pays en développement appliquant des normes environnementales moins strictes à leurs producteurs. Face à la montée du « label écologique » et au risque que cela se transforme en barrière aux importations en provenance des pays en développement, il est indispensable d'entreprendre un effort d'harmonisation des standards de production au niveau international, d'autant plus que l'on assiste aujourd'hui au foisonnement de ces labels souvent sans aucun contrôle de la part des organismes officiels nationaux. Par exemple en Suisse, seul le label Bourgeon utilisé pour les produits biologiques est garanti par la Confédération. ${ }^{25}$ La fixation de normes peut avoir des effets négatifs pour les pays qui ne sont pas en mesure de les respecter en raison de leur stade de développement ou du manque de savoir-faire ou de moyens techniques. Il est dès lors

${ }^{22}$ CNUCED, Sustainable Development : Trade and Environment - The Impact of Environment-related Policies on Export Competitiveness and Market Access, TD/B/41(1)4, 1994.

${ }^{23}$ Cf. P. Uimonen, « Trade Rules and Environmental Controversies During the Uruguay Round and Beyond ", The World Economy, vol. 18, n² 1, 1995.

${ }^{24}$ Cf. S. Vaughan, « Trade and Environment : Building a Revolutionary Framework », Industry and Environment, janviermars 1994.

${ }^{25}$ Cf. M. Egger et J. Forster, « Les mesures de coopération au développement », dans P. Dembinski, J. Forster et J. de Melo (eds.), Effets de l'Uruguay Round sur les pays en développement, Mandat de l'OFAEE, Genève, 1995 (vol. 4, sec. B). 
primordial de prendre en compte la réalité des pays en développement et en transition au moment de l'élaboration de ces normes, notamment en les associant à ce processus. Cette tâche pourrait être confiée par exemple à l'OMC.

En Suisse aussi, les consommateurs sont de plus en plus conscients des liens entre commerce, développement et environnement et veulent être informés sur l'origine et les conditions de productiòn de biens provenant des pays en développement. ${ }^{26} \mathrm{C}$ 'est la raison pour laquelle la Confédération collabore aux travaux multilatéraux visant à une transparence aussi large que possible sur l'origine et les conditions de production de certains produits éco-sensibles tels que les bois tropicaux. Elle étudie ainsi l'introduction de labels facultatifs, par exemple pour le bois tropical produit dans des conditions de développement durable. Aussi, dans le cadre de mesures nationales en faveur de l'environnement et de limitations apportées au commerce, la Suisse veille à éviter des mesures qui peuvent avoir des effets négatifs sur les économies des pays en développement et, à long terme, aussi sur leur environnement. La Suisse reconnaît que les pays en développement qui, pour des raisons financières et techniques, ne sont pas en mesure d'atteindre des normes écologiques adéquates méritent un soutien pour améliorer leurs méthodes de production et de transformation. Une étude récente sur les effets de l'Uruguay Round sur les pays en développement a proposé un certain nombre de mesures concrètes que la Confédération pourrait adopter en relation avec la gestion des labels écologiques et le risque d'éco-protectionnisme. ${ }^{27}$

\subsection{ACCORDS INTERNATIONAUX SUR LES MATIĖRES PREMIÈRES}

Les dégâts infligés à l'écosystème dans les pays en développement sont souvent liés aux échanges internationaux des matières premières. La plupart des pays producteurs manquent de moyens nécessaires pour investir dans la préservation et la reconstitution de l'environnement. En raison de la vive concurrence qui règne sur les marchés internationaux de matières premières, il est impossible de superposer au prix une somme qui permettrait l'adoption de méthodes de production meilleures du point de vue écologique. Il est par conséquent virtuellement impossible de réaliser sur des marchés de matières premières non organisés l'intégration des externalités liées à l'environnement.

Les accords internationaux sur les matières premières sont parmi les rares instances internationales qui puissent à l'échelle mondiale en organiser et contrôler le prix, la production et la consommation. Il est possible de transformer ces accords pour assurer un relèvement du prix, et ainsi l'adoption de méthodes de production non dommageables pour l'environnement. ${ }^{28} \mathrm{Ce}$ relèvement du prix peut en principe prendre la forme d'une taxe à l'exportation ou d'une surtaxe douanière à l'importation. La première formule demande des mesures complémentaires et une bureaucratie d'audit pour empêcher certains pays d'échapper aux contraintes du système et les pays les moins développés de payer leurs importations plus cher. L'autre formule, celle d'un droit de douane supplémen-

\footnotetext{
${ }^{26}$ Voir à ce propos « Rapport du Conseil fédéral sur les relations Nord-Sud de la Suisse dans les années 90 (Lignes directrices Nord-Sud) ", p. 14.

27 Cf. P. Dembinski et al., op. cit., 1995.

${ }^{28}$ Cf. H. Kox, «Integration of Environmental Externalities in International Commodity Agreements », World Development, août 1991.
} 
taire à l'importation dans les pays acheteurs, paraît préférable. Sous cette forme peuvent être obtenues les ressources financières liées à la production et à la consommation qui alimenteront un fonds international permettant le financement, dans les régions productrices, de projets de préservation et de reconstitution de l'environnement.

\subsection{TRANSFERT DE TECHNOLOGIE}

Jusqu'à présent, le transfert de technologie, d'ampleur somme toute modeste, a cherché à amorcer la croissance économique au Sud sans motivation écologique explicite. Or, ce type de transfert revêt une importance particulière en tant que vecteur de propagation des modes de production plus écologiques dans le monde en développement et ceci pour deux raisons principales. D'abord, la réalisation des objectifs de développement durable nécessite des améliorations sensibles de productivité, lesquelles ne peuvent être assurées qu'à travers l'usage d'une technologie de production plus performante. Ensuite, la technologie est de plus en plus considérée comme un agent majeur de gestion environnementale. Notons que les technologies susceptibles d'être transférées des pays industrialisés occidentaux vers les pays en développement incluent non seulement les biens d'équipement, mais également les spécifications de design, le capital humain et surtout la capacité de développement et de gestion du renouveau technologique. Ce dernier élément s'avère être d'une importance capitale pour le succès d'un transfert. En effet, le transfert de technologie ne se résume pas uniquement à l'introduction d'une nouvelle technologie. Il doit être accompagné du transfert du savoir-faire nécessaire pour l'adaptation autonome et continue de cette technologie.

L'acquisition et la maîtrise des technologies écologiquement justes ont été reconnues par la CNUED comme étant cruciales pour atteindre l'objectif du développement durable, d'où la nécessité d'une action concertée au niveau international visant à promouvoir ou faciliter le transfert international de telles technologies. Jusqu'ici l'effort international le plus significatif concernant le transfert de technologies écologiquement justes a été déployé dans le cadre du programme " Pour une Production Plus Propre » ( Cleaner Production ») mis sur pied dès 1990 par le Programme des Nations Unies pour l'environnement (PNUE). Ce programme, qui se trouve encore à ses balbutiements, rencontre actuellement des difficultés sérieuses liées à l'absence de financement sur le plan international et local. ${ }^{29}$

En plus des contraintes financières, les réglementations du commerce international, les lois sur les brevets et les dispositions relatives aux licences de fabrication sont souvent citées comme étant les principaux obstacles au transfert de technologies écologiquement justes vers les pays en développement.$^{30} \mathrm{Or}$, les faits ont démontré que le relâchement des restrictions liées à la propriété intellectuelle ou aux licences ne conduit pas nécessairement à un transfert de technologie significativement plus volumineux. En effet, on a assisté à plusieurs reprises à l'incapacité des pays en développement à faire plein usage de l'information technolo-

${ }^{29}$ Cf. S. Hafez, « Financing Cleaner Production in Developing Countries », Industry and Environment, Décembre 1994, pp. 75-76.

${ }^{30}$ Pour une discussion des problèmes liés à la propriété intellectuelle dans le transfert de technologie, voir E. Mansfield, Intellectual Property Protection, Foreign Direct Investment and Technology Transfer, Washington, Banque mondiale, 1994. 
gique se trouvant dans le domaine public. C'est ainsi que la capacité d'assimilation de la technologie, plutôt que les barrières aux transferts, est considérée comme le principal obstacle à l'acquisition de technologies plus propres par les pays en développement. ${ }^{31}$ De plus en plus, il apparaît que le succès des programmes internationaux de coopération en matière de transfert de technologies écologiquement justes dépend de la volonté des gouvernements des pays récepteurs d'introduire ou non des réformes internes visant à faciliter l'adoption et le développement de ces technologies. ${ }^{32}$

Pour la Suisse, le transfert de technologie fait partie des objectifs de l'aide bilatérale et multilatérale même si les transferts facilités par l'aide publique suisse au développement n'ont pas toujours été favorables à l'environnement. ${ }^{33}$ Parmi les autres instruments pouvant être utilisés dans ce but, on peut mentionner la Garantie contre les risques à l'exportation (GRE). En effet, depuis 1981, la GRE doit tenir compte des politiques et principes de développement lorsqu'il s'agit d'exportations vers les pays les plus pauvres. On note que la part des exportations de machines dans les garanties atteint $80 \%$. Le potentiel de transfert de technologie écologiquement juste inhérent à cet instrument est donc loin d'être négligeable. ${ }^{34}$

\subsection{LIEN AVEC LES POLITIQUES NATIONALES DE L'ENVIRONNEMENT AU NORD}

Lorsqu'il s'agit de problèmes environnementaux de caractère global, on peut établir un lien entre les politiques nationales de l'environnement des pays développés et l'effort d'assainissement dans les pays en développement. On peut illustrer ce lien en prenant comme exemple le cas d'émission de gaz à effet de serre. Actuellement, certains pays occidentaux, se voyant incapables de tenir leurs engagements consistant à stabiliser d'ici à l'an 2000 leurs émissions de $\mathrm{CO}_{2}$ aux niveaux de 1990, ont proposé la « mise en œuvre conjointe » (joint implementation) des mesures d'assainissement. La mise en œuvre conjointe permet à un pays de remplir en partie ses engagements en finançant des actions sur le territoire d'un pays en développement ou en transition. Cette proposition se base sur le fait que la même réduction dans les émissions nocives peut être obtenue à un coût nettement inférieur dans ces pays par rapport aux pays développés. Quelques projets-pilote de ce type existent déjà sur la base des accords bilatéraux (par exemple, entre la Norvège et le Mexique). La mise en œuvre conjointe peut constituer un argument de poids pour faire accepter aux pays développés les plus réticents des réductions supplémentaires d'émission de gaz à effet de serre.

En Suisse, le projet de la taxe $\mathrm{CO}_{2}$ présenté et mis en consultation en 1994 a suscité une forte opposition de la part de l'économie privée, laquelle craignait que l'introduction d'une telle taxe de manière solitaire n'affaiblisse la compétitivité de l'appareil de production helvétique. Face à cette opposition, la décision défi-

${ }^{31}$ Cf. C. Juma et M. Sagoff, « Policies for Technology », dans J. C. Dooge et al. (eds.), An Agenda of Science for Environment and Development into the 21 st Century (Cambridge, Cambridge University Press, 1992).

${ }^{32}$ Pour une discussion approfondie des réformes requises voir C. Juma, « Promoting International Transfer of Environmentally Sound Technologies : The Case for National Incentive Schemes », Green Globe Yearbook, 1994, pp. 137-148.

${ }^{33}$ Par exemple, la rallonge d'un crédit mixte accordé à la Thaïlande en 1993 a été fortement critiquée car elle complétait le financement d'une centrale électrique très polluante. Cf. Annuaire Suisse-Tiers Monde, 1995, p. 133.

${ }^{34}$ Voir à ce propos L. Kébir, Mesures gouvernementales stimulant les transferts de technologie et développement durable : le cas de la Suisse, mémoire de DESMAP, Université de Genève, 1996. 
nitive à ce sujet a été reportée jusqu'à la fin de ce siècle. De toute façon, il ne serait question de l'introduction d'une telle taxe que si les émissions ne pourraient pas être réduites par des mesures volontaires. La nouvelle Loi sur l'énergie, actuellement en préparation, devrait rappeler l'objectif de la stabilisation des émissions de $\mathrm{CO}_{2}$ pour l'an 2000 et fixer des objectifs à atteindre en 2005, 2010 et 2020. Quel que soit l'instrument utilisé pour atteindre ces objectifs, l'effort d'assainissement pèsera de plus en plus lourd sur l'économie de la Suisse, connue comme étant l'un des pays de l'OCDE à appliquer les normes environnementales les plus sévères. Dans ce contexte, la mise en œuvre conjointe paraît particulièrement intéressante et mériterait d'être soutenue activement par la Suisse dans les futures négociations internationales. Si une taxe $\mathrm{CO}_{2}$ devait néanmoins être introduite après l'an 2000 , on pourrait prévoir d'y adjoindre un mécanisme de crédit d'impôt à destination des entreprises suisses effectuant un effort de réduction d'émissions dans les pays en développement.

\subsection{FINANCEMENT DE L'ASSAINISSEMENT ÉCOLOGIQUE}

Jusqu'ici nous avons esquivé le problème le plus épineux en rapport avec l'environnement et le développement, à savoir celui du financement de l'effort d'assainissement écologique dans les pays en développement, dans la mesure où nous avons surtout évoqué des instruments ne nécessitant pas de moyens de financement accrus de la part des pays développés, avec l'exception notable du transfert de technologie. Or, la question du financement se trouve au centre de cette problématique et constitue souvent l'obstacle majeur à la mise en œuvre des politiques environnementales.

Prenons, à titre d'exemple, l'Agenda 21 (voir ci-dessus). Le chapitre de ce catalogue de mesures qui a donné lieu aux débats les plus âpres concerne le financement des programmes convenus. Le document adopté souligne que seuls la croissance économique, l'ouverture des échanges commerciaux et les investissements permettront de dégager les ressources nécessaires. Les fonds devront être fournis par les pollueurs (par le biais de redevances et de «droits à polluer » négociables), le secteur commercial et des sources volontaires et publiques. Or, compte tenu de la lenteur des pays industrialisés occidentaux à introduire les instruments économiques de politique environnementale sur le plan interne, on peut imaginer que la mobilisation des sources de financement précitées est au mieux très hypothétique dans les pays en développement, au moins à court et moyen terme. Pour mettre en œuvre rapidement des programmes environnementaux dans ces pays, il faut surtout se fonder sur le financement concessionnel.

Le financement concessionnel prend d'abord la forme d'aide publique au développement. Lors du Sommet de la Terre à Rio, les pays industrialisés ont réaffirmé leur engagement d'atteindre l'objectif de $0,7 \%$ du PNB fixé par les Nations Unies. Actuellement, seuls les Pays-Bas et les pays scandinaves ont déjà atteint cet objectif. Certains pays, tels que l'Allemagne et la France, se sont engagés à le faire d'ici à l'an 2000 ; d'autres, tels que les Etats-Unis (qui en sont actuellement à $0,2 \%$ seulement), ont refusé de souscrire à cet objectif. La Suisse, quant à elle, avait prévu de faire passer le budget alloué à la coopération au dévelop- 
pement à $0,4 \%$ du PNB avant la seconde moitié des années $90 . .^{35} \mathrm{Vu}$ l'état critique des finances fédérales, cet objectif n'a pas pu être réalisé. Sans augmentation sensible de l'enveloppe budgétaire consacrée à l'aide publique au développement, des projets environnementaux peuvent difficilement être réalisés sans compromettre les autres objectifs visés par les programmes nationaux d'aide.

Actuellement, un fonds international d'une certaine envergure visant à aider les pays en développement à faire face aux problèmes nationaux d'environnement fait cruellement défaut. Quant aux projets écologiques aux retombées mondiales, leur principale source de financement internationale est le Fonds pour l'environnement mondial (FEM), créé en 1990 avec une dotation initiale de 1,5 milliard de dollars sous forme d'engagements. Administré conjointement par la Banque mondiale, le PNUD et le PNUE, le FEM finance des projets visant à combattre le réchauffement de la planète, la destruction de la biodiversité, la pollution des eaux internationales et l'appauvrissement de la couche d'ozone. Il est prévu d'élargir le champ d'action du FEM pour inclure des problèmes de dégradation des sols, tels que la désertification et la déforestation. Durant la phase de lancement du FEM, la Suisse a fourni $4 \%$ du fonds, ce qui constitue une contribution plus que proportionnelle. Cela témoigne de l'intérêt qu'elle porte à l'intégration des pays en développement dans le processus de résolution des problèmes écologiques planétaires. La Suisse participe activement aux négociations lancées en vue de la restructuration du FEM. L'objectif est de mieux l'adapter aux dispositions des conventions.

\subsection{ENDETTEMENT ET ENVIRONNEMENT}

La CNUED a attiré l'attention du monde sur le fait que la demande pour le financement des projets de conservation de la nature dans les pays du tiers monde est directement liée à leur sous-développement ainsi qu'à leur niveau d'endettement. Le débat sur la préservation des forêts tropicales est une parfaite illustration de ce dilemme. Préoccupés par des problèmes environnementaux comme le réchauffement de la planète, les pays développés appellent à des mesures visant à maintenir l'équilibre du climat terrestre, celui-ci étant menacé surtout par leurs propres émissions excessives de $\mathrm{CO}_{2}$. Pour les pays en développement, la protection de la nature ne constitue pas toujours la priorité absolue. Afin de promouvoir le développement économique et social et de réunir les devises nécessaires au service de leur dette externe, ils sacrifient leurs forêts sur l'autel de l'exploitation minière, du tourisme, de l'agriculture et de l'industrie du bois largement au-delà du seuil de reconstitution naturelle de cette précieuse ressource. Les experts estiment qu'au rythme actuel d'exploitation de ces forêts, elles auront entièrement disparu avant l'an 2020.

Les échanges dette/protection de la nature (debt-for-nature swaps) constituent un instrument ingénieux pour financer les projets environnementaux dans les pays en développement tout en allégeant leur dette externe. Cet instrument consiste en l'achat sur le marché secondaire des titres de la dette par un organisme international de la protection de l'environnement, puis à convertir cette dette en monnaie nationale du pays en développement en vue de sa mise à dis-

${ }^{35}$ Cf. Message du 15.5.1991 concernant l'adhésion de la Suisse aux institutions de Bretton Woods. 
position pour la réalisation des programmes environnementaux dans le pays en question.

Si jusqu'ici seuls une vingtaine d'accords de ce type ont pu être conclus dans une dizaine de pays, plusieurs facteurs peuvent expliquer cet échec relatif. ${ }^{36}$ Premièrement, un échange dette/protection de la nature fait intervenir plusieurs acteurs : une organisation internationale de protection de la nature (souvent une ONG), une banque internationale, les agences gouvernementales du pays concerné, la Banque centrale du pays et une organisation nationale de protection de la nature. L'implication d'autant d'agents différents ne simplifie certainement pas la conclusion d'un accord. Deuxièmement, le succès d'un tel accord dépend de manière cruciale de la bonne volonté des gouvernements des pays récepteurs. Or, ces gouvernements craignent souvent la perte de leur souveraineté nationale au profit des ONG internationales. Le statut politique des organisations nationales de protection de l'environnement diffère également d'un pays à l'autre. Troisièmement, les Banques centrales des pays impliqués hésitent souvent à convertir des sommes importantes de devises en monnaie nationale par crainte d'effets inflationnistes. Ce problème pourrait toutefois être résolu en transformant la dette non pas en monnaie mais en obligations nationales, dont les intérêts serviraient à financer les projets environnementaux. Quatrièmement, si l'escompte sur la dette peut parfois atteindre $85 \%$, les échanges dette/protection de la nature doivent tout de même être financés d'une manière ou d'une autre, dans la mesure où les organisations internationales de protection de la nature sont des organismes à but non lucratif. On retombe donc de nouveau sur le problème du financement déjà abordé ci-dessus. Cela dit, le financement pourrait provenir de l'effacement pur et simple de la dette, ce qui contribuerait à améliorer l'image internationale du donateur, qu'il s'agisse d'un pays ou d'une banque internationale. Depuis 1991, la Confédération procède chaque année à une remise de dette d'environ 6070 millions de francs en faveur des pays en développement. Les fonds en question proviennent d'un crédit de 700 millions de francs (dont 400 millions réservés pour le désendettement), ouvert en 1991 à l'occasion du $700^{\mathrm{e}}$ anniversaire de la Confédération. Il est à regretter que ces fonds ne soient pas utilisés dans le cadre des échanges dette/protection de la nature.

\section{CONCLUSIONS}

La protection de l'environnement fait partie intégrante du développement. Sans une bonne protection de l'environnement, il n'y aura pas de développement durable. Sans développement, il n'y aura pas de ressources suffisantes pour effectuer les investissements qui s'imposent, et donc pas de protection de l'environnement. Or, si l'importance de la prise en compte de la dimension environnementale dans la conception des programmes de développement est aujourd'hui largement reconnue, les moyens mis en œuvre par les pays développés et les organisations internationales sont loin d'être à la hauteur des ambitions telles qu'elles ressortent des déclarations de bonnes intentions qui font l'unanimité lors des conférences internationales.

\footnotetext{
${ }^{36}$ Cf. O. Schmid-Schönbein, « Debt-for-Nature Swaps : Funding Conservation in Third World Countries », Economic and Financial Prospects, $n^{9}$ 6, décembre-janvier 1992.
} 
On ne peut s'empêcher d'être pessimiste quant à l'avenir des problèmes environnementaux de caractère local ou régional dans les pays en développement, pourtant d'importance capitale pour le développement notamment à travers leurs effets sur la santé des populations. On comprend aisément que ces problèmes reçoivent beaucoup moins d'attention auprès de l'opinion publique des pays développés que les problèmes liés aux pollutions à effet planétaire. Il est donc plus difficile de dégager les ressources nécessaires pour réaliser ce type d'assainissement dans les pays en développement. Ceci peut être expliqué en partie par le ralentissement de la croissance dans les pays développés, ce qui les rendrait moins «altruistes », mais également par le fait que ces pays ne se sentent pas « responsables » de ce type de pollution dans les pays en développement. Quant aux problèmes liés aux pollutions à effet global, les perspectives sont moins sombres. D'abord, comme l'exemple récent des mesures prises contre les CFC le montre, la communauté internationale, les pays développés en tête, sait parfois prendre les mesures nécessaires pour éviter une catastrophe d'ampleur planétaire, une fois que le seuil du danger a été clairement franchi et à condition qu'il y ait une certaine convergence de vue chez les scientifiques. D'autant plus s'il s'agit d'un domaine tel que le réchauffement de la planète, où une part prépondérante de la responsabilité de la pollution incombe au monde industrialisé.

Le problème du financement se trouve au cœur de la problématique d'assainissement écologique dans le tiers monde. Une chose est claire : il ne faut pas attendre de miracle du côté des pays en développement eux-mêmes. La préoccupation environnementale constitue rarement une priorité dans ces pays. Certains aspects de la protection de la nature comme la biodiversité sont souvent considérés comme du « luxe de riche ». Il faut donc trouver du financement à l'extérieur. Il y a d'abord l'aide publique traditionnelle, bilatérale et multilatérale. Or, aujourd'hui le montant de cette aide est insuffisant pour qu'on espère réaliser de cette manière des projets à caractère environnemental en plus de projets ordinaires de développement. Tout au plus, on peut essayer de soumettre les projets réalisés dans le cadre de l'aide au développement à des analyses d'impact environnemental, en vue d'en minimiser les effets néfastes, voire de trouver des synergies. Ensuite, il y a des organismes internationaux créés dans un but spécifique de protection de la nature et les ONG de caractère écologique. Ceux-ci doivent également être financés d'une manière ou d'une autre. La conversion de la dette du tiers monde en fonds pouvant servir à la protection de l'environnement est une solution qui n'a pas été pleinement exploitée jusqu'à maintenant. Une autre possibilité, sujette à controverse, serait de financer ces organismes par une taxe internationale prélevée sur des matières premières polluantes ou sur les opérations financières. Enfin, il y a la possibilité, hélas très peu explorée, de mettre à contribution des agents privés. Pour cela, il faut bien entendu qu'il y ait un mécanisme d'incitation. On peut utiliser la fiscalité à cet effet, par exemple en affranchissant d'impôt les dépenses d'investissement à caractère écologique effectuées par les entreprises des pays développés dans le tiers monde. Mais on peut aussi négocier des contrats de parrainage avec les entreprises qui seraient ainsi en mesure d'améliorer leur image de marque grâce à leur appui financier à un projet écologique tel que, par exemple, la constitution d'un parc national dans un pays en développement. 